\title{
Study of strain localization and energy dissipation in metals based on infrared thermography
}

\author{
by O.A. Plekhov, A. Fedorova, A. Kostina and I. Panteleev
}

Institute of continuous media mechnics UB RAS, 1 ak. Korolev str., 614013, Perm, Russia, poa@icmm.ru

\begin{abstract}
This work is devoted to theoretical and experimental study of energy balance in titanium alloys under plastic deformation and failure. It is shown that infrared thermography can give a unique opportunity to investigate the thermodynamics of plastic deformation and failure of metals. The energy balance in titanium alloy OT-4 was studied in smooth samples under quasi-static loading and at fatigue crack tip under crack propagation. Extending previous results of the research group in Perm, we coupled the experimental investigation of temperature evolution with a statistical description of the mesodefect ensemble. It allowed us to propose a thermodynamic internal variable model of heat dissipation in metals.
\end{abstract}

\section{Introduction}

The experimental and theoretical study of energy balance under irreversible deformation has an extensive bibliography. The importance of this issue was originally shown by J. H. Lambert in 1779 in his statement concerning the energy similarity of mechanical and thermal failure processes of solids. The review of experimental works devoted to the methods of stored energy investigation in the material under plastic deformation is available in [1].

To analyze the thermodynamic characteristics of deformation processes in solids, it is necessary to take into account the fact that the plastic deformation work is converted into two parts: heat energy caused by the movement and annihilation of defects at various structural levels, and hidden (stored) energy of plastic deformation accumulated in the elastic fields of defects.

During the last decades the enhanced ability to detect temperature evolution during mechanical testing leads to a great interest in the application of infrared (IR) thermography to study heat dissipation process caused by defect evolution under plastic deformation and failure. The theoretical and experimental study of this problem can be useful for explanation of many actual problems of mechanics of solids such as problem of study of cold work, investigation of damage to fracture transition and crack propagation process, investigation of applicability of crack tip closure concept, validation of linear failure mechanics formula to the investigation of fatigue cracks propagation under different loading conditions and so on.

This work is devoted to theoretical and experimental study of energy dissipation in titanium alloy OT-4 (Russian notation) under deformation and failure based on infrared thermography data. The developed algorithms allow us to calculate the heat source distribution on the specimen surface. It gives us the possibility to calculate the stored energy rate under quasistatic loading and estimate the heat source evolution at crack tip under fatigue crack propagation.

The extension of previous theoretical results of the research group in Perm, we coupled the experimental investigation of temperature evolution with a statistical description of the mesodefect ensemble. It allowed us to develop a thermodynamic internal variable model of heat dissipation in metals. The proposed model allows us to simulate the energy balance in metals under quasistatic loading.

\section{Materials and conditions of experiments}

The experimental study of temperature evolution was carried out on plane specimens of titanium alloy OT-4. The chemical composition of the material is presented in the Table 1. The mechanical properties were determined during standard quasistatic test, the fatigue properties were estimated by the handbook. The mechanical properties are shown in the Table 2.

Table 1: The chemical composition of titanium alloy(\%).

\begin{tabular}{|l|l|l|l|l|l|l|l|l|l|}
\hline $\mathrm{Al}$ & $\mathrm{Zr}$ & $\mathrm{Si}$ & $\mathrm{Fe}$ & $\mathrm{O}$ & $\mathrm{H}$ & $\mathrm{N}$ & $\mathrm{C}$ & $\mathrm{Mn}$ & $\mathrm{Ti}$ \\
\hline 3.68 & 0.3 & 0.12 & 0.3 & 0.15 & 0.012 & 0.05 & 0.1 & 1.16 & other \\
\hline
\end{tabular}

Table 2: The mechanical properties.

\begin{tabular}{|l|l|l|l|l|}
\hline $\begin{array}{l}\text { Modulus } \\
\text { elasticity }\end{array}$ & Yltimate stress & Fatigue limit & Fracture toughness \\
\hline $64 \mathrm{GPa}$ & $600 \mathrm{MPa}$ & $900 \mathrm{MPa}$ & $460 \mathrm{MPa}$ & $75.6 \mathrm{MPa} \sqrt{\mathrm{m}}$ \\
\hline
\end{tabular}


The specimens were manufactured from a titanium sheet $3 \mathrm{~mm}$ thick. The specimen gage part was $20 \mathrm{~mm}$ width $100 \mathrm{~mm}$ long. The specimens for crack propagation study with size 3×55×200 mm were weakened by three holes to initiate fatigue crack at the specimen centre. The fatigue crack (about $10 \mathrm{~mm}$ ) was initiated at the initial stage of the experiment by high amplitude cyclic loading. Then the load was decreased to slow down the rate of crack propagation. It is allow us to perform a detailed analysis of the heat generation processes at the crack tip.

The test conditions comply with the conditions of the experiment described in [2]. The temperature evolution was recorded by infrared camera FLIR SC 5000 . The spectral range of the camera is 3-5 mm. The maximum frame size is $320 \times 256$ pixels; the spatial resolution is $10^{-4}$ meters. The temperature sensitivity is from $25 \mathrm{mK}$ to $300 \mathrm{~K}$.

The surface of the specimens was polished in several stages by the abrasive paper (at the final stage of polishing the grit size does not exceed $3 \mu \mathrm{m}$ ). Before starting the experiment, the polished surface was covered by a thin layer of amorphous carbon.

\section{Experimental results}

The definition of heat sources evolution requests the calculation of the space and time derivation of noisy temperature field. It leads to the situation when recorded experimental data of the temperature is not enough suitable to calculate the heat dissipation rate.

Specimen motion relative to the infrared camera lens under cyclic loading conditions leads to the problem with processing of experimental infrared data. To get the correct temperature data at a given point on the specimen surface, the original procedure of motion compensation was developed. Compensation of the relative motion was implemented by dint of the algorithm described in details in [2]. The algorithm is based on the selection of marker-zone on the studied surface and searching this area on the surface in each following time steps. It allowed us to determine a displacement of each point on the specimen surface as a time function.

An original procedure of time and space filtering of experimental data allowed us to eliminate the noise caused by influence of environment. Filtration procedures included simultaneous application of the two- dimensional discrete Fourier transform for the space signal and one- dimensional discrete Fourier transform for the time signal on the base of the standard Gaussian kernel. The following equations illustrate the general mathematical interpretation of such filtering

$$
\begin{gathered}
T(x, y)=\int_{-\infty}^{\infty} \frac{1}{(2 \pi)^{2}} \exp \left(-i\left(k_{x} x+k_{y} y\right)\right) \hat{T}\left(k_{x}, k_{y}\right) \hat{f}_{1}\left(k_{x}, k_{y}\right) d k_{x} d k_{y} \\
T(t)=\int_{-\infty}^{\infty} \frac{1}{2 \pi} \exp \left(-i k_{x} t\right) \hat{T}\left(k_{t}\right) \hat{f}_{2}\left(k_{t}\right) d k_{t}
\end{gathered}
$$

where $f_{1}(x, y)=\frac{v^{2}}{\pi} \exp \left(-v^{2}\left(x^{2}+y^{2}\right)\right)$ - Gaussian kernel for space filtering, $f_{2}(\mathrm{t})=\frac{v^{2}}{\pi} \exp \left(-v^{2} \mathrm{t}^{2}\right)-$ Gaussian kernel for time filtering, $\hat{f}_{1}\left(k_{x}, k_{y}\right)$ and $\hat{f}_{2}\left(k_{t}\right)$ are the direct Fourier transforms of the standard Gaussian kernel, $\widehat{T}\left(k_{x}, k_{y}\right)$ and $\hat{T}\left(k_{t}\right)$ are the direct Fourier transforms of the temperature, $x$ and $y$ are spatial coordinates, $t$ is time, $v$ is the filter size.

The initial temperature field obtained directly from the experiment and the result of application of filtering procedure to the experimental data are presented in figure $1 \mathrm{f}$ and figure $1 \mathrm{~b}$ respectively. The processed temperature field (figure 1b) was used to calculate the heat sources field near the fatigue crack tip.

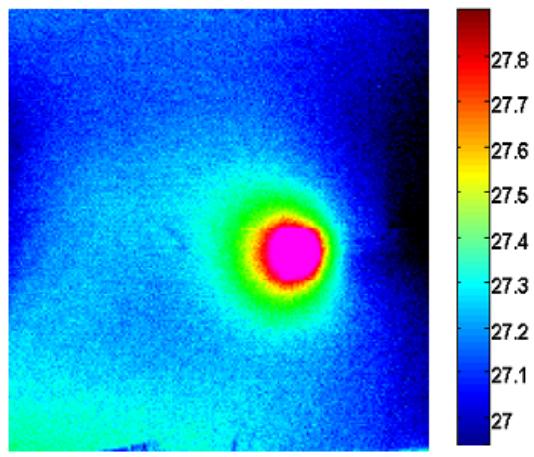

A

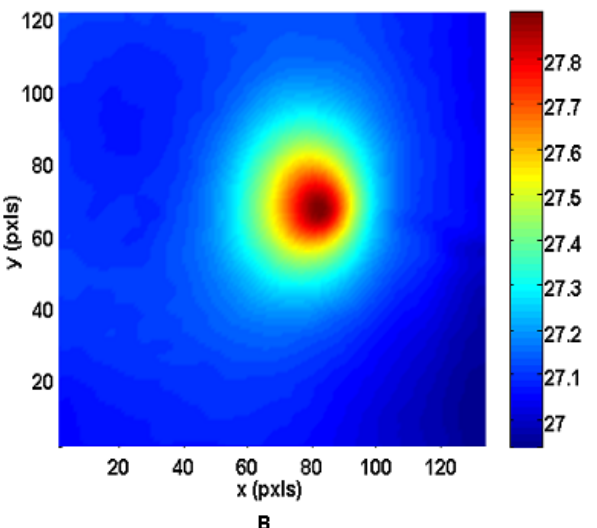

B

Fig. 1 Infrared image before data processing $(A)$ and processed temperature field (B). 
To calculate the specific heat source rate we have used finite difference scheme of the heat conduction equation (3). The forward finite difference scheme was used to evaluate the time derivation. To assess the Laplacian of the temperature function a 2 nd order central finite difference scheme was applied

$$
S(x, y, t)=\rho c\left(\dot{\theta}(x, y, t)+\frac{\theta(x, y, t)-\theta(x, y, 1)}{\tau}\right)-k \Delta \theta(x, y, t),
$$

where $\theta$ - temperature field, $\dot{\theta}$ - time derivation of the temperature field, $\theta(x, y, 1)-$ the initial temperature field of the specimen without loading, $\Delta \theta$ - the Laplacian of the temperature function, $\rho$ - density $\left(4430 \mathrm{~kg} / \mathrm{m}^{3}\right), c-$ heat capacity $(668 \mathrm{~J} /(\mathrm{kg} \cdot \mathrm{K})), k$ - heat conductivity $(11.7 \mathrm{~W} /(\mathrm{m} \cdot \mathrm{K})), \mathrm{S}(x, y, t)-$ unknown specific energy dissipation $\mathrm{rate}\left(\mathrm{W} / \mathrm{m}^{3}\right), r-\mathrm{a}$ constant related to the losses of heat by heat exchange with the surroundings (50 s).

The value of $T$ was determined on the base of the standard analysis of specimen cooling process. This identification procedure consists of two steps. The first one is to measure a temperature evolution during a simple return to room temperature from heterogeneous thermal state. This initial heterogeneous thermal state can be obtained by a local heating of the specimen during a few seconds (by contact, laser and etc.). The second one is to calculate the time derivative and Laplacian of the temperature function provided the heat sources are equal to zero during this return to the room temperature. Then using thermal conductivity equation with a zero heat source the unknown constant $T$ is easy determined.

This procedure allows us to smooth spatial and time heterogeneity. Fig. 2 presents an example of the temperature field under quasi-static tension sample without crack before and after processing.

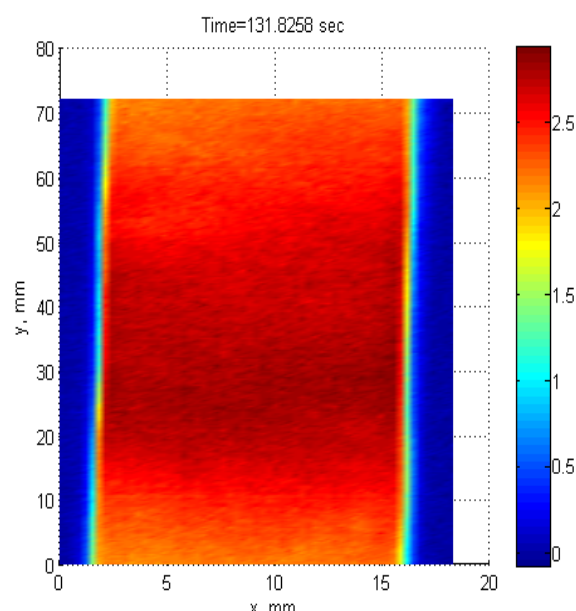

a)

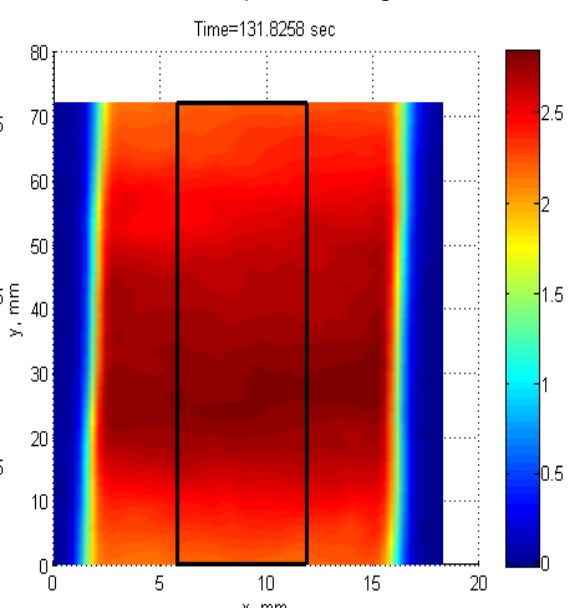

b)

Fig. 2: Temperature range on the surface of specimen before data processing (a) and obtained field of temperature range with zone of interest (b)

Fig. 3 presents field of heat dissipation rate on the small area of specimen surface limited by black rectangle shown in Fig $2 \mathrm{~b}$. The small area for calculation was selected due to sample shape changing during deformation process. The field of heat sources is a little bit heterogeneous even during the second part of plastic deformation.
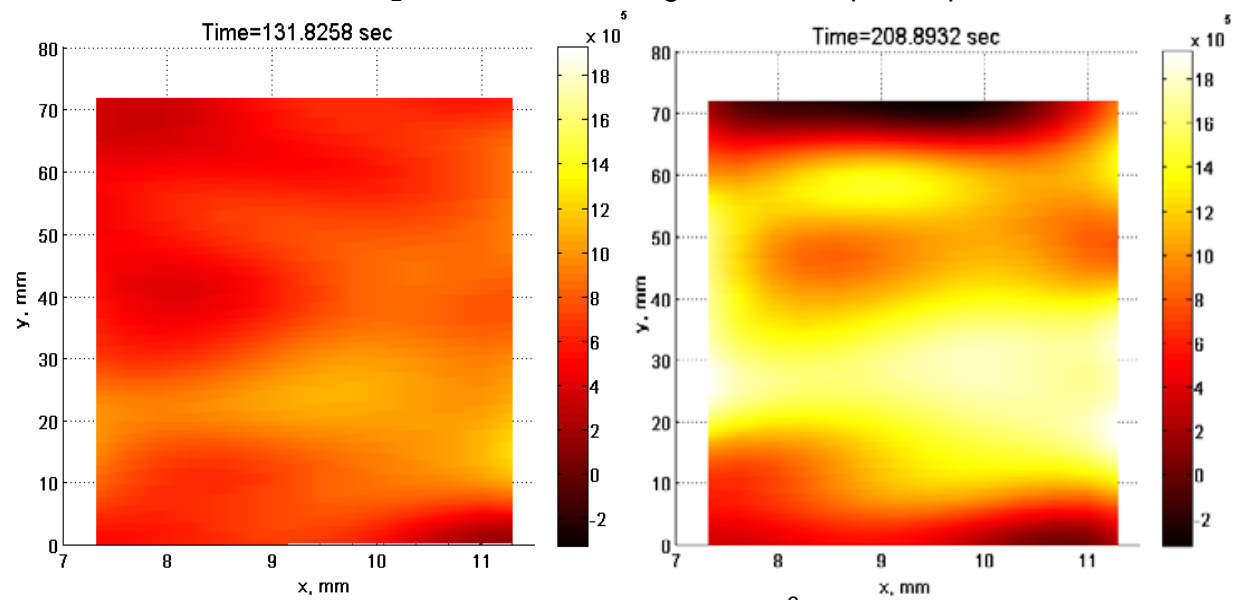

Fig. 3 Experimental data of the heat dissipation rate $\left(\mathrm{W} / \mathrm{m}^{3}\right)$ at the beginning and end of experiment 
The integral heat dissipation in zone of interest can be calculated as

$$
Q(t)=\int_{x 1}^{x 2} \int_{y 1}^{y 2} s(x, y, t) d x d y
$$

where $x_{1}, x_{2}, y_{1}, y_{2}$ are the rectangular coordinates of the interest zone (Fig. 2b).

We suppose that some of the irreversible plastic work contributes to heat generation, while the rest is stored as the energy of crystal defects accompanying plastic deformation, traditionally known as the stored energy of cold work [1]. The goal of this work is to calculate value of the stored energy rate.

Plastic work spend on deformation of the specimen has to be known for calculation of the energy stored in metals during deformation. We obtained plastic work presented in expression (3) as a function of strain rate $V$ and loading force $F(t)$

$$
W_{p}(t)=F(t) V
$$

We calculate stored energy of cold work as difference between plastic work spent on deformation and integral heat dissipation $\left(W_{p}(t)-Q(t)\right)[1]$. Time dependence of these values is shown in Fig. 3.

Ratio $\frac{Q(t)}{W_{p}(t)}$ is usually used for determination of the parameter $\beta$ characterized extent of material damage. The stored energy ratio can be calculated as follow

$$
1-\beta=\frac{W_{p}(t)-Q(t)}{W_{p}(t)}
$$

In Fig. 4 value of $1-\beta$ depending on strain is presented. Value of stored energy decreases in the end of deformation process that means most of plastic work converted into heat and materials prepared to the failure.

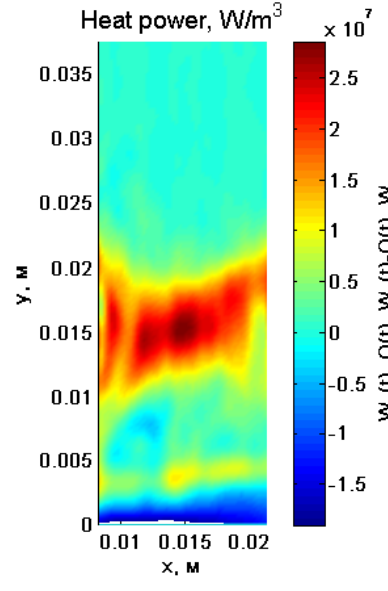

a

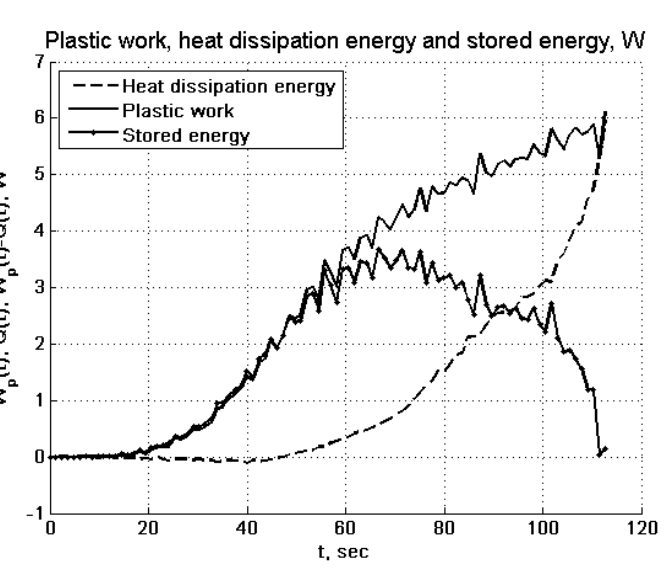

b

Fig. 4 Experimental data for the heat power field close to fracture (a), time dependence of plastic work, heat dissipation energy and stored energy calculated for the smooth specimen (b).

Crack propagation tests were carried out at the stress ratio $\mathrm{R}=-0.03$. Maximal loading applied to the specimen was $307.62 \mathrm{MPa}$, minimal was $-9.35 \mathrm{MPa}$. Before starting of the cyclic experiment the specimen was loaded by the mean value of stress (148.23 MPa) and testing was began from this position.

The specimens were weakened by holes to initiate fatigue crack at the specimen center. The process of crack propagation was studied at $10 \mathrm{~Hz}$ loading frequency and $100 \mathrm{~Hz}$ frame rate of the infrared camera to obtain ten time points with infrared data on one loading cycle and to investigate the evolution of heat dissipation during this cycle. The selected frequency of loading provides conditions close to adiabatic. This condition practically excludes the heat transfer by the convection mechanism from the heat generation area near the crack tip towards the surroundings. But this condition does not exclude the heat transfer by the conduction mechanism from heat zone to cold zone in the area near the crack tip. It is taken into consideration by the corresponding part of equation 1. 


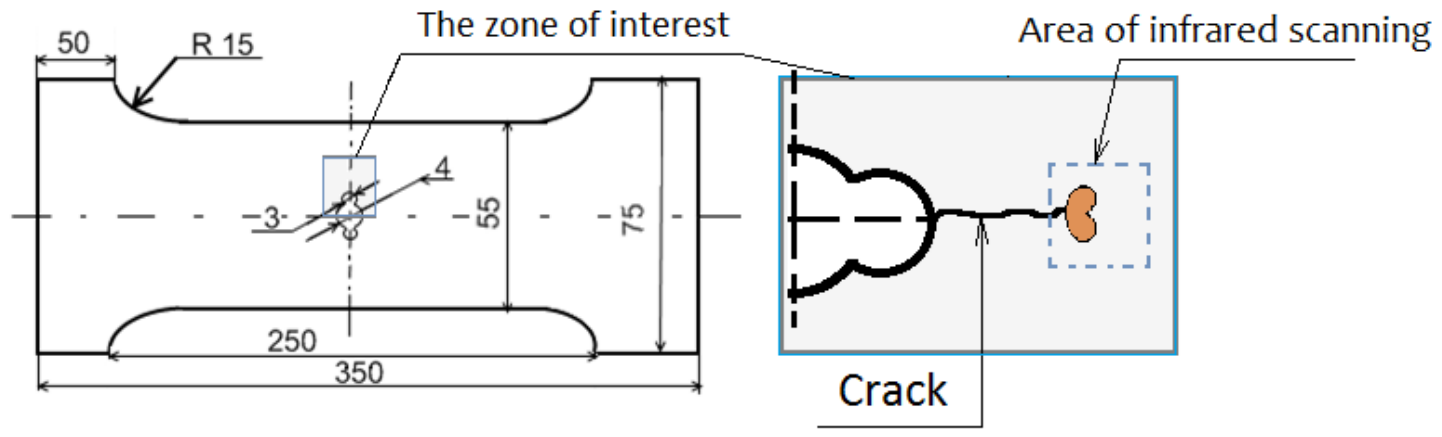

Fig. 1. Geometry of sample (all sizes are in millimeters).

The result of calculations of the heat dissipation field at fatigue crack tip is presented in figure 4 . The heat dissipation data and the time dependence of loading were synchronized by superimposition of the first moment of loading and the first changing in temperature data. We analyzed one cycle of loading highlighted by bold line on the graph. The corresponding cycle on the heat dissipation curve is also marked by bold line. Ten IR frames on one loading cycle were recorded. The numbers from 1 to 10 on the graph indicate these IR frames. The IR camera was registering the temperature not only in one point on the specimen surface but the temperature fields of the area near the crack tip during cyclic loading. After calculations of the heat sources by expression (3) ten fields illustrating the heat dissipation rate were obtained in the crack tip area. The bottom part of figure 4 shows these fields corresponding to the points on the graph from the top part of figure 4.

Two types of the plastic zone appear under cyclic loading conditions. Formation of the monotonic plastic zone is connected with maximal tensile stress in fatigue crack tip area. When the direction of force is changed the compression stresses appears in monotonic plastic zone. Formation of the cyclic plastic zone is connected with significant compression stresses which are appear in fatigue crack tip while in front of the crack the tensile stresses occur. When materials reached the yield stress these compression stresses form cyclic plastic zone. That is why the plastic cyclic zone forms even in the case of tensile loading.

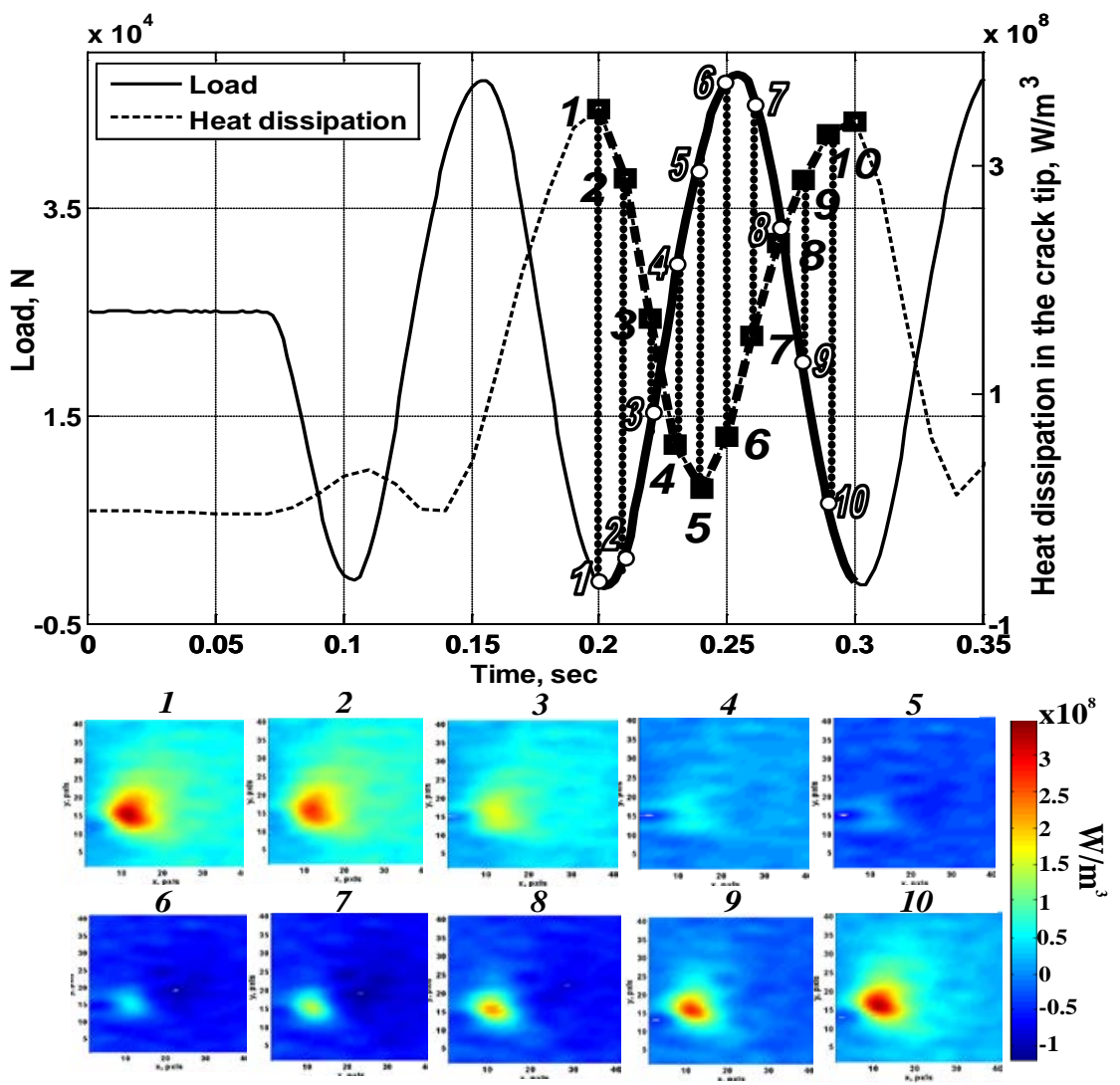

Fig. 5 Evolution of the heat sources field during one cycle of loading.

Analysis of the data presented in figure 4 allows us to divide the heat dissipation rate caused by the evolution of monotonic and cyclic plastic zones. Period from points 6 to 7 approximately correspond to the maximal tensile stresses. The heat sources fields 6-7 illustrate the formation of the monotonic plastic zone. The small heating area (about 0.5- 
1.3.10 $\mathrm{W} / \mathrm{m}^{3}$ ) could be seen on the cold background of the specimen which is under elastic conditions excluding the crack tip where the monotonic plastic zone exists. In period from point 7 to 10 , the unloading is started. All surface of specimen is unloaded excepting the crack tip where the compressional stresses appear due to the previous plastic deformation in this point. The cyclic plastic zone in crack tip area is formed. The compressional stresses near the crack tip are accompanied by intensive heat dissipation. That is why on the heat dissipation fields 7-10 the heat spot is made more intensive near crack tip. In point 10-1 the loading is about zero but compressional stresses in the crack tip reach the maximum value. Because of it, on the heat sources field 10 and 1 the maximum heat dissipation (about 3-3.5.10 ${ }^{8}$ $\mathrm{W} / \mathrm{m}^{3}$ ) is observed. In period from point 1 to point 6 the specimen is under tension that is accompanied by cooling of the specimen surface together with the crack tip area. In this period, near the crack tip only the monotonic plastic zone exists but on the background of the previous intensive heating this monotonic plastic zone is revealed only in the point 6 . All processes are repeated on the next cycles of loading.

Observed heat dissipation coincides with the theoretical conception of the different types of plastic zone formation. The calculation of stored energy map requests the plastic work distribution at crack tip. An example of calculation of stored energy map based on the consideration of HHR-solution is presented in [4].

\section{Theoretical model and numerical simulation}

To describe the energy balance in solid under plastic deformation and failure we have to describe and simulate the structure evolution of the solid. It is now well known that real metals have complex structure, which is a hierarchy of different levels. Under deformation process the structural evolution observed at all scale levels and leads to irreversible deformation and destruction. To develop a model of defect evolution under plastic deformation we have to choose the basic physical level of description of the material microstructure and to describe the geometry of the elementary defects. One of the possible descriptions of defect kinetics is the statistical model of defect ensample. This model has to take into account the stochastically properties of defect initiation, their nonlinear integration and link between microplasticity and damage accumulation properties. form [5]:

Typical mesoscopic defects (mesoshifts) are described as the symmetrical tensor $\tilde{S}$, which has the following

$$
\tilde{s}=\frac{1}{2} S(\bar{n} \bar{l}+\bar{l} \bar{n}),
$$

where $\bar{l}$ is a unit normal to the shear plane, $\bar{b}$ is a unit vector in the shear direction, $S$ is a shift intensity.

Defect density tensor that coincides with the strain caused by defects is defined as:

$$
\tilde{p}=n<\tilde{s}>
$$

where $n$ is a defect density.

The distribution function of defects can be represented in the following form [5]

$$
W=Z^{-1} \exp (-E / \theta)
$$

where $E$ is the energy of the defect, $Z$ is the normalizing factor, $\theta$ is the effective temperature factor responsible for the system susceptibility.

Averaging procedure lets us to obtain the self-consistency equation between micro and macro parameters in the following form:

$$
\tilde{p}=N \int \tilde{s} W(\tilde{s}) d \tilde{s}
$$

The solution of the equation (5) was proposed in [12]. Based on these results we can introduce a phenomenological model of the quasistatic process. Full strain rate can be represented as the sum of three components: elastic strain rate $\dot{\tilde{\varepsilon}}^{e}$, plastic strain rate $\dot{\tilde{\varepsilon}}^{p}$ and strain rate caused by defects $\dot{\tilde{p}}$ :

$$
\dot{\tilde{\varepsilon}}=\dot{\tilde{\varepsilon}}^{e}+\dot{\tilde{\varepsilon}}^{p}+\dot{\tilde{p}}
$$

Elastic strains are defined by linear Hooke's law:

$$
\dot{\sigma}_{0}=K \dot{\varepsilon}_{0}^{e}
$$




$$
\dot{\tilde{\sigma}}_{d}=2 G \dot{\tilde{\varepsilon}}_{d}^{e}
$$

where $K$ - isotropic elastic modulus, $G$ - elastic shear modulus, $\sigma_{0}$-spherical stress tensor, $\tilde{\sigma}_{d}$ - deviator of stress, $\varepsilon_{0}^{e}$-spherical elastic strain tensor, $\tilde{\varepsilon}_{d}^{e}$ - deviator of elastic strain.

Dissipation function for a medium with defects can be represented in the following form [13]:

$$
\tilde{\sigma}: \dot{\tilde{\varepsilon}}^{p}+\left(\tilde{\sigma}-\rho \frac{\partial F}{\partial \tilde{p}}\right): \dot{\tilde{p}}-\bar{q} \cdot \frac{\nabla T}{T} \geq 0
$$

where $F$ is a free energy, $\rho$ - density, $\bar{q}$ - heat flux vector, $T$ - temperature.

Dividing the thermal and mechanical problems and basing on the Onsager principle, we can obtain from (11) constitutive equations for calculating kinetics of plastic and structural strains:

$$
\begin{aligned}
\dot{\tilde{\varepsilon}}^{p} & =\Gamma_{\sigma} \tilde{\sigma}+\Gamma_{p \sigma}\left(\tilde{\sigma}-\rho \frac{\partial F}{\partial \tilde{p}}\right), \\
\dot{\tilde{p}} & =\Gamma_{p}\left(\tilde{\sigma}-\rho \frac{\partial F}{\partial \tilde{p}}\right)+\Gamma_{p \sigma} \tilde{\sigma},
\end{aligned}
$$

The kinetic coefficients $\Gamma_{\sigma}, \Gamma_{p}$ and $\Gamma_{p \sigma}$ have the following form:

$$
\begin{gathered}
\Gamma_{\sigma}=\frac{1}{\tau_{\sigma}} \frac{1}{1+\operatorname{Exp}\left(-\frac{|\sigma|-S_{c}}{a_{1}}\right)}, \\
\Gamma_{p}=\frac{1}{\tau_{p}} \frac{1}{1+\operatorname{Exp}\left(-\frac{H\left(|\sigma|,|p|, \delta, p_{c}, \sigma_{c}\right)-S_{y}}{a_{2}}\right)}, \\
\Gamma_{p \sigma}=\frac{1}{\tau_{p \sigma}},
\end{gathered}
$$

where $\tau_{\sigma}, \tau_{p}, \tau_{p \sigma}$ - characteristic relaxation times, $|\sigma|$ - stress intensity tensor, $S_{c} a_{1}, a_{2}$ - material constants, $S_{y}$ - yield stress, $|p|$ - intensity of $\tilde{p}, \quad p_{c}, \sigma_{c} \quad$ - scaling factors, $H\left(|\sigma|,|p|, \delta, p_{c}\right)=|\sigma|-2 \mu \sigma_{c}\left[\delta(f+1)|p| p_{c}-|p| p_{c}\right]$. - material function (it can be considered as "degree of system nonequilibrium").

It is supposed that thermodynamic force $\tilde{\sigma}-\rho \frac{\partial F}{\partial \tilde{p}}$ can be written as:

$$
\tilde{\sigma}-\frac{\partial F}{\partial \tilde{p}}=\left[\frac{1}{\delta}\left(\frac{\tilde{\sigma}}{2 G \sigma_{c}}+\frac{\tilde{p}}{p_{c}}\right)-\left(f\left(\frac{|p|}{p_{c}}\right) \frac{p_{c}}{|p|}+1\right) \frac{\tilde{p}}{p_{c}}\right],
$$

where $f(|p|)$ denotes a power function for modeling of nonlinear hardening process:

$$
f\left(\frac{|p|}{p_{c}}\right)=k\left(\frac{|p|}{p_{c}}\right)^{a},
$$

$k$ is a scaling factor, $a$ is the exponent. hardening.

Equations (8)-(10) and (12)-(15) represent a closed system for a plastically deformed solid with nonlinear

From the first thermodynamic law, we can obtain the expression for calculation the $\beta$ parameter in the following form: 


$$
\beta=\frac{\dot{Q}^{p}}{\dot{W}^{p}}=1-\frac{\left[\rho \frac{\partial F}{\partial \tilde{p}}-\rho T \frac{\partial^{2} F}{\partial T \partial \tilde{p}}\right]: \dot{\tilde{p}}}{\tilde{\sigma}:\left(\dot{\tilde{\varepsilon}}^{p}+\dot{\tilde{p}}\right)},
$$

where $Q^{p}$ - inelastic contribution to the heat, $W^{p}$ - plastic work. Under isoentropy conditions it has the form:

$$
\beta=\frac{\dot{Q}^{p}}{\dot{W}^{p}}=1-\frac{\rho \frac{\partial F}{\partial \tilde{p}}: \dot{\tilde{p}}}{\tilde{\sigma}:\left(\dot{\tilde{\varepsilon}}^{p}+\dot{\tilde{p}}\right)} .
$$

Based on the proposed model we have modeled a quasistatic loading of a smooth specimen under strain rate $\dot{\varepsilon}=4.3 \cdot 10^{-3} C^{-1}$. The simulation of mechanical properties is presented in figure 6 . Numerical simulation of the considered process was carried out in the finite element package Simulia Abaqus 6.13. All using modulus have licenses. Eight-node linear brick elements were used for the simulation. The above explained model was applied for the material behavior description using the subroutine UMAT. Arrays of material constants, strain, strain increments and the time step passed as input data to the procedure. Increments of the stress tensor components and increments of the defect density tensor components are determined from the system of constitutive equations. Values of these components at the next time step are defined as the sum of the values on the previous step and the appropriate increment.

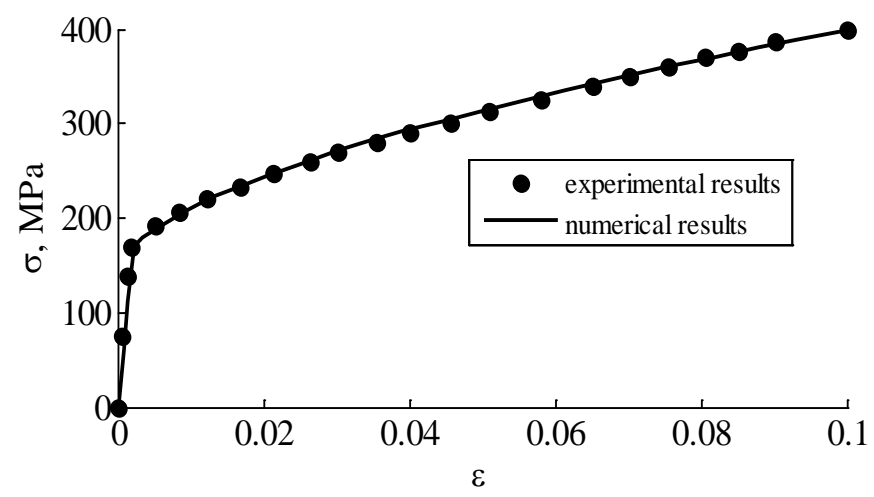

Fig.6 Experimental and simulated stress-strain curves

The three-dimensional results of the considered process simulation are presented in figures 2 (steel specimen) and 3 (armco iron specimen). Almost uniform distribution of the (1- $\beta$ ) parameter in the gauge part is observed in both cases.

The modeling results of the energy storage rate evolution are shown in figure 7 . This dependence has a maximum which agrees with the experimental data [7]. According to the structural investigations, on the initial stage of a plastic deformation there is observed a preparation of the material structure associated with the initiation of new structural defects. This leads to a growth of the energy storage rate. When a strain reaches some level, the dominant processes are growth, movement and annihilation of structural defects that leads to a growth of a dissipated energy portion.

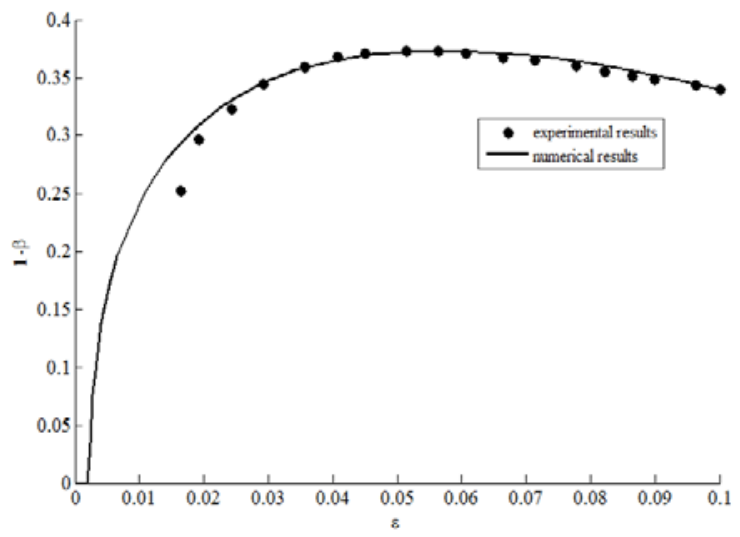

Fig. 7 Value of the 1- $\beta$ parameter (steel) versus strain. Numerical and experimental results 


\section{Conclusion}

This work is devoted to experimental study of energy balance in metals under plastic deformation and failure. The calculation of stored energy rate can give us a unique opportunity to describe the structure evolution of metals and develop new effective criteria of material fracture. It is shown that infrared themography allows us to investigate the problem of energy dissipation under plastic deformation. This technique can be considered both as tool for verification of existing constitutive models of plastic deformation and effective monitoring technique.

The extension of previous theoretical results of our research group, we coupled the experimental investigation of temperature evolution with a statistical description of the mesodefect ensemble. It allowed us to develop the original thermodynamic internal variable model of heat dissipation in metals. Based on the model there were considered numerical tensile quasistatic experiments of steel and the value of stored energy rate was calculated. It was shown that on the initial stage of the plastic deformation the storage energy rate increases reaching its maximum value, which is equivalent to the defect density increase in the material. On the final stage of the plastic deformation the energy storage rate decreases. This can be connected with a predominance of dissipative processes. The obtaining results are in a quantitative agreement with the experimental.

\section{Acknowledgement}

The work is partially supported by grants RFBR (№14-01-00122) and grant for leading Russian scientific school (HШ2590.2014.1).

\section{REFERENCES}

[1] Bever M.B., Holt D.L., Tichener A.L. "The stored energy of cold work". Prog. Mat. Sci. 17 (1973), p. 1.

[2] Fedorova A., Bannikov M., Plekhov O. "A study of the stored energy in titanium under deformation and failure using infrared data". Fracture and structural integrity, 21 (2012), p. 46.

[3] Plekhov O., Naimark O. "Theoretical and experimental study of energy dissipation under strain localization in metals" Journal of Appl. Mech. and Tech. Phys., vol. 50, n1, (2009), p. 1.

[4] Fedorova A., Bannikova M., Terekhina A., Plekhov O. Heat dissipation energy under fatigue based on infrared data processing // QIRT v.11, issue 1,( 2013) 2-9 pp.

[5] Naimark, O.B. Collective properties of defects ensemble and some nonlinear problems of plasticity and failure. Phys. Mesomech.(2003) 4:45-72. 